\title{
Axially linear slopes of composition for "delta" crystals
}

\author{
P. Gille ${ }^{1}$, M. Hollatz, H. Kleessen, M. Schenk * \\ Institut für Kristallographie und Materialforschung, Fachbereich Physik, Humboldt-Universität zu Berlin, Invalidenstrasse 110, \\ D-10115 Berlin, Germany
}

(Received 4 October 1993; manuscript received in final form 28 December 1993)

\begin{abstract}
"Delta" crystals are solid solutions of miscible materials with large lattice parameter differences which contain high concentration gradients in one direction (parallel to a lattice plane strongly diffracting X-rays). The system $\mathrm{GaSb}-\mathrm{InSb}$ has been chosen as suitable for study. By means of a "gradient projection method", the growth of nearly linear composition profiles with relatively steep slopes of the lattice parameter (up to $(\Delta a / \bar{a}) / \Delta z=8.3 \% \mathrm{~cm}^{-1}$ ), adjustable by the temperature gradient, have been performed. However, the grown ingots were not monocrystalline due to the use of too high a growth rate.
\end{abstract}

\section{Introduction}

$\mathrm{X}$-ray optics, in contrast to the optics of visible light, suffers from the negligibly small refraction of X-rays in solids. In an original paper, Smither [1] has published concepts for focusing X-rays by means of diffraction through the use of crystals having a laterally changed lattice parameter. From Fig. 1 it is evident that to convert divergent radiation into a narrow bundle of parallel $\mathrm{X}$-rays, the reflecting crystal must be bent and must additionally have a continuously changing lattice parameter. This change can be obtained either by using a temperature gradient in a homogeneous crystal or by the use of a defined gradient of

\footnotetext{
* Corresponding author.

1 Present address: Institut für Werkstoffwissenschaften VI, Universität Erlangen-Nürnberg, Martensstrasse 7, D-91058 Erlangen, Germany.
}

composition. The first method was investigated by Smither and yielded an effect of the order of $\Delta a / \bar{a} \approx 10^{-3}$ per $\mathrm{cm}$ for temperature differences of some hundreds of kelvin, which is much too small for X-ray optics: several percent per $\mathrm{cm}$ are generally needed. The second approach has not been practically pursued to date.

Generally, in crystal growth the opposite problem is treated: how to prepare a homogeneous crystal from a multicomponent system. In mixed crystal systems with a wide separation between solidus and liquidus the segregation coefficients are far from unity, so that most conventional crystal growth methods produce inhomogeneous component distributions. These types of uniaxial distribution functions are well described in the literature and depend on the overall material balance and the special conditions of matter transport. All these component distributions are markedly non-linear. 


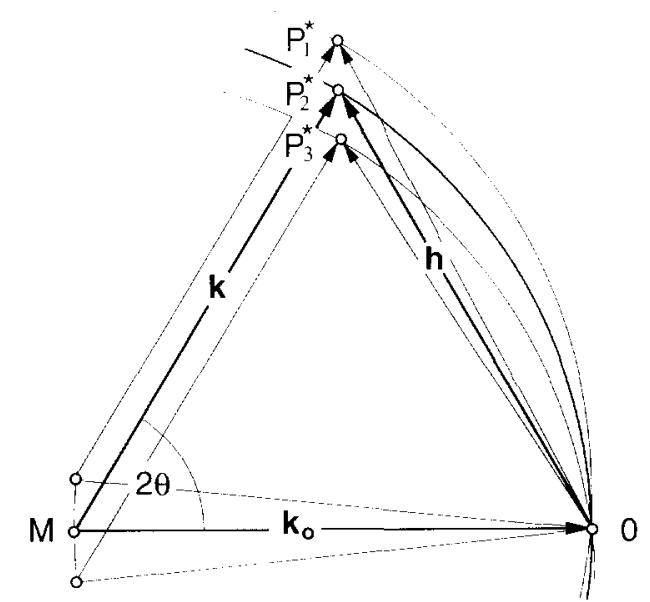

Fig. 1. Conversion of divergent monochromatic radiation into a narrow bundle of parallel X-rays by diffraction at a single crystal. Schematic representation in the reciprocal space (Ewald construction) where $\boldsymbol{k}_{1}$ is the direction of the incident beam, $k$ the direction of the diffracted $\mathrm{X}$-rays, and $\boldsymbol{h}$ a reciprocal lattice vector that locally changes its length (i.e. the lattice parameter laterally changes) as well as its direction (i.e. the crystal is bent). $\mathrm{M}$ : centres of the reflecting spheres with radius $1 / \lambda ; \lambda$ : wavelength of the used radiation; $O$ : origin of the reciprocal lattice: $\mathbf{P}^{*}$; reciprocal lattice points.
There have been a few reported attempts to continuously vary the composition of a mixed crystal in a programmed way. Johnston and Tiller [2] analysed the required variation of the effective distribution coefficient in order to produce a particular solute distribution in the solid. The predictions were tested by controlling the width of the momentum boundary layer in the liquid using an applied rotating magnetic field. In the travelling heater method (THM) of solution growth of graded II-VI alloys, Triboulet et al. [3] used feed rods composed of wedge-shaped binary ingots which provided a source material of continuously varying composition.

In the present paper we will show that relatively steep and axially linear concentration profiles can produce linear lattice parameter changes using a novel growth method involving solid solutions of materials having sufficiently different lattice parameters. Initially, the solid solution system $\mathrm{GaSb}-\mathrm{InSb}$ was investigated. It is characterized by strong segregation effects during crystallization from the melt. Following a suggestion of Bradaczek et al. [4], we designate crystals contain-
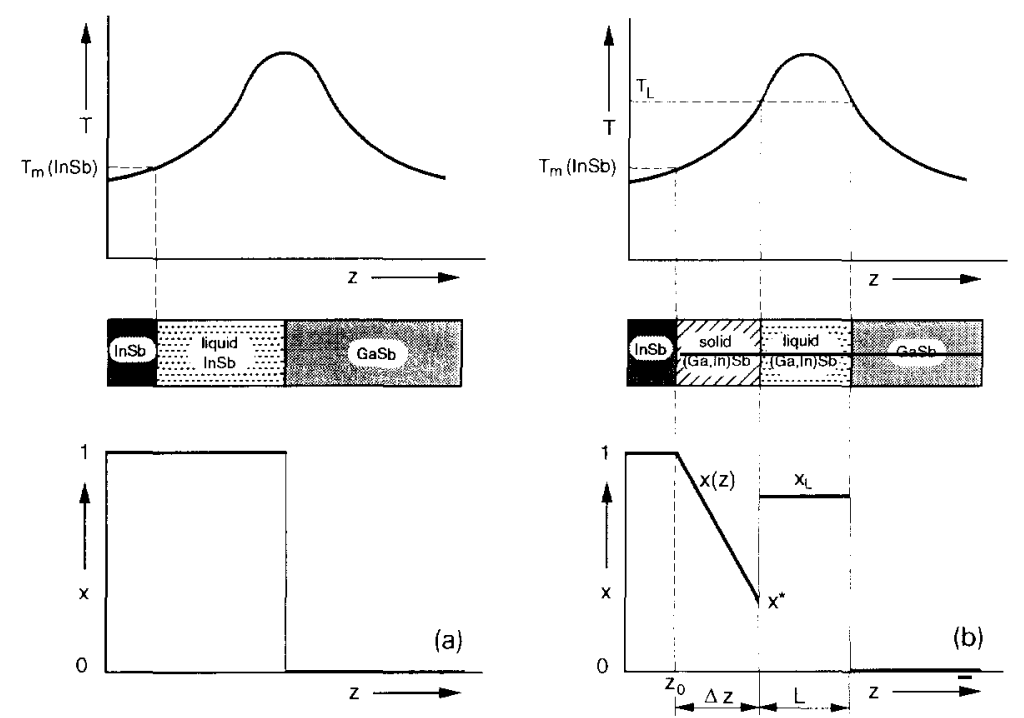

Fig. 2. Schematic representation of the growth method ("gradient projection method"): (a) early stage and (b) final stage of the process to crystallize linearly graded $\mathrm{Ga}_{1-x} \mathrm{In}_{x} \mathrm{Sb}$ alloys from the binary compounds. 
ing marked lattice parameter gradients caused by changes of the chemical composition as "delta" crystals.

\section{The solid solution system GaSb-InSb}

$\mathrm{GaSb}$ and $\mathrm{InSb}$ are completely miscible and crystallize in the zincblende structure, space group $\mathrm{F} \overline{4} 3 \mathrm{~m}$. Their room temperature lattice parameters, $a$, are $a_{\mathrm{GaSb}}=0.609593 \mathrm{~nm}$ and $a_{\mathrm{InSb}}=$ $0.647937 \mathrm{~nm}$, respectively, a $6.01 \%$ difference, related to the average value $\bar{a}$. Gong et al. [5] studied the dependence of the lattice parameter a on composition $x$ in $\mathrm{Ga}_{1-x} \mathrm{In}_{x} \mathrm{Sb}$ and found $\mathrm{a}$ linear relation that obeys Vegard's law. Thus,

$a(x)=0.609593+0.038344 x \mathrm{~nm}$

is used for calculating lattice parameter gradients from profiles of mole fraction $x(z)$.

The relatively low melting points of $T_{\mathrm{m}}(\mathrm{GaSb})$ $=712^{\circ} \mathrm{C}$ and $T_{\mathrm{m}}(\mathrm{InSb})=525^{\circ} \mathrm{C}$, and modest vapour pressures of 0.1 and $0.004 \mathrm{~Pa}$, respectively, facilitate the growth procedure. The mechanical properties [6] should allow the necessary treatment of the crystals (cutting, polishing, bending), so that the transition of stresses and strains due to the strong lattice parameter gradients into plastic deformation [7] may be avoided. For all estimates of segregation phenomena we used the quasi-binary phase diagram established by Blom and Plaskett [8].

\section{Crystal growth}

The principle of the growth method is to start using significant non-equilibrium conditions between the two binary constituents and allow the system to approach steady state. As it is shown in Fig. 2, ingots of pure InSb and $\mathrm{GaSb}$ are placed in the temperature field of a zone heater with their touching front faces at the maximum of the temperature profile. If the absolute temperature is chosen to greatly exceed the InSb melting point but not that of $\mathrm{GaSb}$, the starting conditions are characterized by a partially molten InSb ingot and unchanged GaSb (Fig. 2a). Since this type of

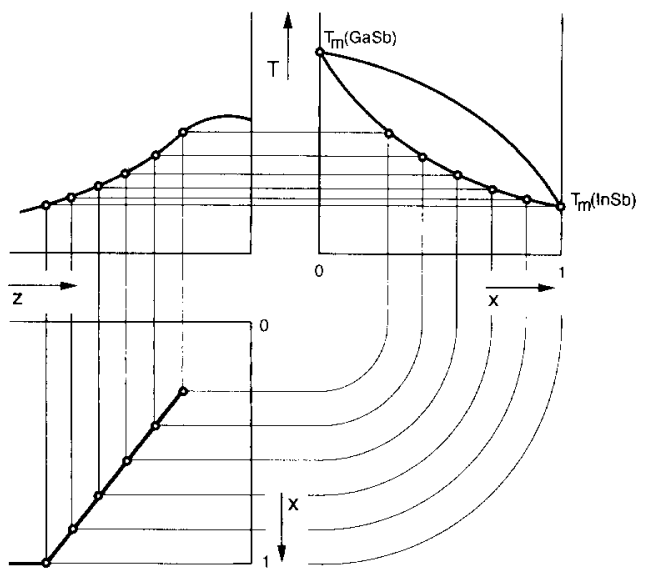

Fig. 3. Schematic $T(x)$ phase diagram of GaSb-InSb that projects a given axial temperature curve $T(z)$ on the resulting composition profile $x(z)$ in the mixed crystal $\mathrm{Ga}_{1-x} \mathrm{In}_{x} \mathrm{Sb}$.

molten zone is not in equilibrium with $\mathrm{GaSb}$, but is capable of dissolving the higher melting point material, $\mathrm{GaSb}$ will move along the concentration gradient and reach the InSb phase boundary. There, the liquid becomes supersaturated with respect to the actual temperature and crystallizes with an alloy composition according to the solidus of the pseudo-binary phase diagram. The crystal growth process will cease when the molten zone of composition $\mathrm{Ga}_{1-x_{1}} \mathrm{In}_{x_{1}} \mathrm{Sb}$ in contact with solid $\mathrm{Ga}_{1-x^{*}} \operatorname{In}_{x^{*}} \mathrm{Sb}$ and $\mathrm{GaSb}$, respectively, is symmetrically located within the axial profile (Fig. 2b) ${ }^{\# 1}$. Thus, both the starting and the final situation are well determined by the temperature field and the phase diagram. It is difficult to calculate or control the rate of the crystal growth process. However, the composition profile $x(z)$ of the $\mathrm{Ga}_{1-x} \mathrm{In}_{x} \mathrm{Sb}$ crystal grown on the remaining InSb, which can act as a seed, is not influenced by this growth rate or matter transport conditions, but only by the temperature profile projected onto the axial position by the slope of the solidus line

\footnotetext{
\#1 Of course, no thermodynamic equilibrium exists between the ternary liquid $\mathrm{Ga}_{1-x} \mathrm{In}_{x_{\mathrm{L}}} \mathrm{Sb}$ and pure GaSb. However, when the molten zone is fully saturated with GaSb and cannot dissolve additional material, the result is the creation of a microscopically steep concentration gradient within the solid.
} 
(Fig. 3). Hence this approach is called the "gradient projection method" (GPM).

From particle balance, starting with a totally symmetric arrangement, the final zone position is characterized by the expression

$\int_{z_{11}}^{z_{11}+\Delta z}[1-x(z)] \mathrm{d} z=\left(x_{1}-\frac{1}{2}\right) L$

where $L$ is final length of the molten zone, $x_{L}$ is mole fraction of $\mathrm{InSb}$ in the final molten zone and $\Delta z$ is length of the grown mixed crystal, which becomes

$$
\left(1-x^{*}\right) \Delta z=\left(2 x_{\mathrm{L}}-1\right) L
$$

for a linear concentration profile with $x^{*}$ being the final mole fraction.

Thus, although a high concentration gradient, which is needed for the delta crystal application, would only require a strong temperature dependence of the solidus but not a wide separation between liquidus and solidus, the latter is necessary for an efficient ratio of the length of the grown material to the residual zone length. Hence GPM is restricted to mixed crystal systems with marked segregation effects, not only to the system under investigation.

With the aid of projections like the one shown in Fig. 3, the axial component distribution can be determined from a given temperature field. In the same way, it is possible to find the necessary temperature curve for a desired concentration profile. Assuming an axially linear temperature

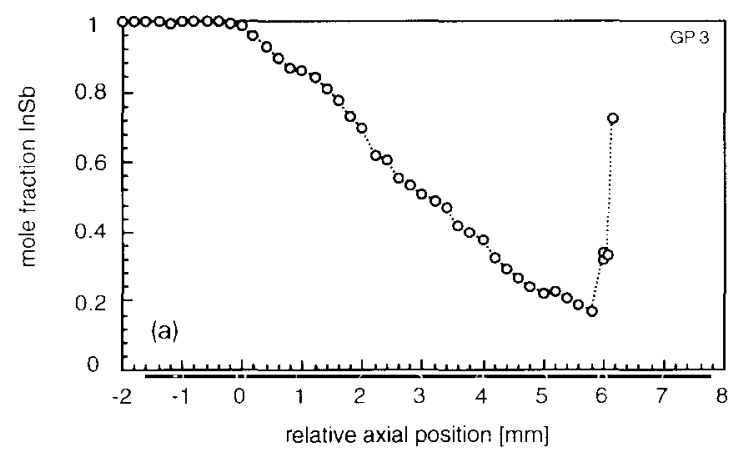

field, only a linear slope of the solidus would result in a constant chemical gradient. Thus, a curved temperature profile such as the low-temperature part of $T(z)$ in Fig. 3 is needed for typical phase diagrams. Such profiles are easily realized with simple zone heating facilities at temperatures lower than the inflection point of the axial profile.

\section{Experimental procedure}

Two different horizontal ring furnaces were used for the GPM experiments. The effective lengths of the temperature regions were about 4 and $8 \mathrm{~cm}$, respectively. The ampoules were rotated during the experiments at 30 to $60 \mathrm{rpm}$ in order to ensure a radial symmetry of the temperature distribution. The actual temperature profiles in the ampoule were measured using a specially designed ampoule with a thermocouple contained in a small silica tube which was inserted into an $\mathrm{InSb}$ ingot. For mechanical reasons the ampoule did not rotate during the measurement. The recorded axial temperature distributions were symmetric with respect to the centre of the furnaces.

$5 \mathrm{~N}$ grade elements were used for the synthesis of stoichiometric InSb and $\mathrm{GaSb}$ ingots in silica ampoules which had the same inner diameter as the crystal growth ampoules $(15 \mathrm{~mm})$. The ingots

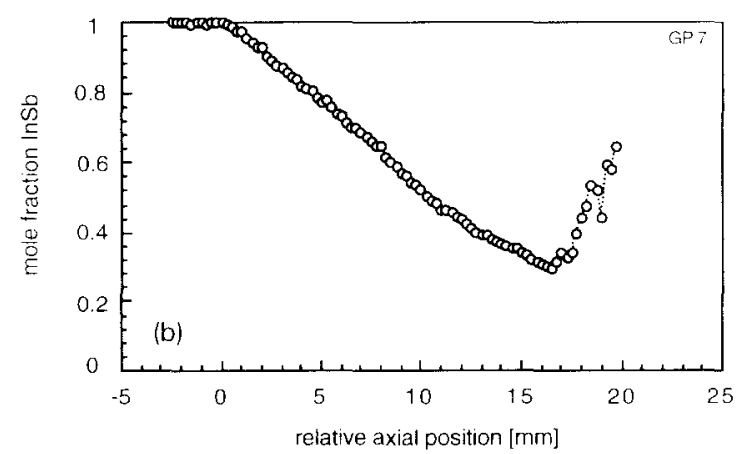

Fig. 4. Axial distributions of mole fraction, $x$, along $\mathrm{Ga}_{1-x} \mathrm{In}_{x} \mathrm{Sb}$ crystals grown by GPM in (a) a steep temperature profile (4 cm long heater, GP 3) and (b) a lower temperature gradient ( $8 \mathrm{~cm}$ long heater, GP 7). EPMA measurement, standard deviation $\Delta x= \pm 2 \times 10^{-3}$. 
were sawn into $40 \mathrm{~mm}$ lengths and the touching faces lapped. After a final cleaning, the ingots were put close together into the ampoule, which was sealed after evacuation to about $10^{-3} \mathrm{~Pa}$. The growth experiments lasted up to seven days. In some experiments, a single-crystalline InSb seed was used. To investigate whether the growth rate with GPM was too fast for stable growth, the ampoule geometry described above was modified in some experiments. A cylinder containing a radial capillary was inserted between the InSb and GaSb ingots. The cylinder was made from silica or steel.

After growth, the ampoules were dissolved in hydrofluoric acid. The ingots were cut along the growth axis, and the faces were lapped and polished for electron probe microanalysis (EPMA) measurements. The diameter of the sample area excited by the electron beam was in the order of 1-2 $\mu \mathrm{m}$. Longitudinal mole fraction distributions were measured by independently monitoring In $\mathrm{K} \alpha$ and $\mathrm{Ga} \mathrm{K} \alpha$ lines on the growth axis and near the periphery of each crystal. A comparison of both concentration profiles enabled isoconcentration curves to be drawn. They may be regarded as isotherms during growth. Lapped surfaces were etched in mixtures of $\mathrm{HF}, \mathrm{HNO}_{3}$, and $\mathrm{H}_{2} \mathrm{O}$ for several seconds in order to reveal the grains.

\section{Results}

All experiments showed qualitatively similar results. The grown $\mathrm{Ga}_{1-x} \mathrm{In}_{x} \mathrm{Sb}$ solid solutions are polycrystalline with grains of some hundreds of $\mu \mathrm{m}$ in size, whether or not a monocrystalline seed was used. All concentration profiles are approximately linear. Fig. 4 shows typical examples of axial concentration profiles of crystals grown in the two types of furnaces used, one having a very high temperature gradient $(4 \mathrm{~cm}$ long heater) and the other a much lower one ( 8 $\mathrm{cm}$ long heater). While the average concentration gradient of crystal GP 3 is as high as $\Delta x / \Delta z=1.4$ $\mathrm{cm}^{-1}$, which is more than sufficient for delta crystal applications, the gradient of crystal GP 7 is $\Delta x / \Delta z=0.43 \mathrm{~cm}^{-1}$. The corresponding changes of the lattice parameter amount to
Table 1

Comparison of experimental results of typical GPM growth runs

\begin{tabular}{lllll}
\hline $\begin{array}{l}\text { Crystal } \\
\text { No. }\end{array}$ & $\Delta x$ & $\begin{array}{l}\Delta z \\
(\mathrm{~cm})\end{array}$ & $\begin{array}{l}(\Delta a / \bar{a}) / \Delta z \\
\left(\mathrm{~cm}^{-1}\right)\end{array}$ & $\begin{array}{l}\Delta T / \Delta z \\
\left(\mathrm{~K} \mathrm{~cm}^{-1}\right)\end{array}$ \\
\hline GP 3 & 0.821 & 0.58 & 0.083 & 170 \\
GP 7 & 0.703 & 1.65 & 0.024 & 40 \\
GP 9 & 0.700 & 1.30 & 0.030 & 53
\end{tabular}

$\Delta x$, maximum mole fraction difference; $\Delta z$, graded crystal length; $(\Delta a / \bar{a}) / \Delta z$, average lattice parameter gradient; $\Delta T / \Delta z$, theoretical average temperature gradient.

$(\Delta a / \bar{a}) / \Delta z=8.3 \% \mathrm{~cm}^{-1}$ and $2.4 \% \mathrm{~cm}^{-1}$ for crystals GP 3 and GP 7 , respectively (Table 1). Isoconcentration lines deduced from EPMA measurements along central and peripheral tracks are plane or slightly concave with respect to the solid depending on the maximum temperature and the location of the grown region relative to the axial furnace position. The concentration profiles resulting from the final molten zone depend on the conditions of cooling at the end of the growth process.

The polycrystallinity of all samples can be attributed to a high degree of constitutional supercooling which results in dendritic growth. Preliminary experiments using a cylinder containing a capillary in order to slow down the matter transport in the liquid and, thus, to reduce the growth rate, resulted only in a slightly coarser grain structure of a few $\mathrm{mm}^{2}$. The axial concentration profile is also almost linear and the average gradient amounts to $\Delta x / \Delta z=0.54 \mathrm{~cm}^{-1}$, corresponding to a relative lattice parameter gradient of $3.0 \% \mathrm{~cm}^{-1}$ (see Table 1, crystal GP 9).

\section{Discussion}

By means of the gradient projection method it was possible to grow $\mathrm{Ga}_{1-x} \mathrm{In}_{x} \mathrm{Sb}$ solid solutions with nearly linear concentration profiles. The different gradients of mole fraction can be qualitatively explained by the axial temperature profiles measured in similar ampoules that were filled with pure InSb only. Changes in the temperature gradient with the two types of furnaces and variations of the heating power are well projected 


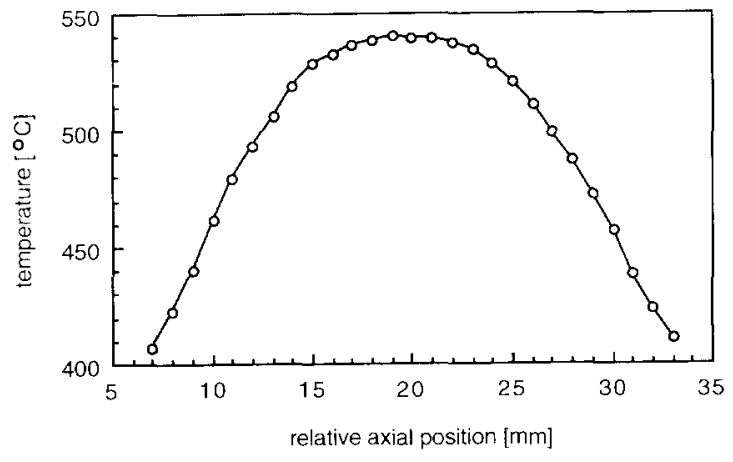

Fig. 5. Characteristic axial temperature profile of the short furnace used, e.g., in experiment GP 3. Measurement in a pure InSb ingot with maximum temperatures about $100 \mathrm{~K}$ lower than used in the GPM experiments.

onto the concentration profiles, as expected from theory. However, the average temperature gradients that must be assumed to explain the resulting gradients of concentration (see Table 1) are approximately twice the measured ones. Regarding the higher thermal conductivity in the melt and the pure $\mathrm{InSb}$ which is almost completely molten in the temperature range to be measured, this simple measurement is only a guide and exact correspondence cannot be expected. On the other hand, measurements with a temperature maximum only slightly exceeding the InSb melting point yielded axial temperature gradients (Fig. 5) sufficiently high to explain the concentration profiles obtained. However, an extrapolation to the temperatures of the experiments, which were about $100 \mathrm{~K}$ higher, is also somewhat questionable.

The polycrystallinity of all samples grown by GPM is assumed to result from too high a growth rate, much exceeding limits given by the constitutional supercooling criterion. The preliminary experiments using a capillary to reduce the growth rate showed that a delay in matter transport does not influence the formation of steep concentration profiles. Different results in the experiments GP 7 and GP 9, carried out with nominally unchanged thermal conditions, are attributed to the influence of the $6 \mathrm{~mm}$ long steel cylinder between the $\mathrm{InSb}$ and $\mathrm{GaSb}$ ingots in the latter experi- ment. Hence the longitudinal slope of concentration in graded solid solutions and the time dependence of the process can be independently influenced. Nevertheless, capillaries inserted into the ampoule are only one of the approaches, possibly not the best, because of problems with the poor wetting of capillary materials by the melt. Attempts will be made to control the crystal growth rate by limiting the rate of GaSb dissolution. These will include starting the process in a position with the temperature maximum well over the $\mathrm{InSb}$ and with a slow movement of the furnace towards the GaSb ingot accompanied by a simultaneous increase in power supply.

\section{Summary}

Binary or pseudobinary solid solutions from components with high lattice parameter differences are, in principle, appropriate for so-called "delta" crystals which have axially linear latlice parameter changes due to strong concentration gradients. The solid solution system $\mathrm{GaSb}-\mathrm{InSb}$ is a suitable system for this purpose.

We have developed a gradient projection method (GPM) and applied it to $\mathrm{Ga}_{1,-}$ In, $\mathrm{Sb}$ which resulted in nearly linear composition profiles with relatively steep slopes but polycrystalline grain structure. Single-crystalline growth has to be achieved before these graded crystals can be used for the X-ray application considered. While GPM allows control of the axial concentration gradient lower growth rates to meet constitutional supercooling criterion limits must be tried in future.

\section{Acknowledgements}

The authors wish to thank Dr. N. Puhlmann and Mr. R.-A. Noack for the EPMA measurements, Mrs. A.-K. Bluhm for technical assistance, and Professors P. Rudolph, H. Bradaczek and G. Hildebrandt and Dr. W. Uebach for supporting discussions. 


\section{References}

[1] R.K. Smither, Rev. Sci. Instr. 53 (1982) 131.

[2] W.C. Johnston and W.A. Tiller, Trans. AIME 224 (1962) 214.

[3] R. Triboulet, G. Neu and B. Fotouhi, J. Crystal Growth 65 (1983) 262.

[4] H. Bradaczek, G. Hildebrandt and W. Uebach, 12th European Crystallographic Meeting, Moscow 1989, Coll. Abstr. Vol. I, p. 304:
H. Bradaczek, G. Hildebrandt and W. Uebach, 16th IUCr Congress, Beijing, 1993, Coll. Abstr. PS-14.01.08, p. 374.

[5] X.Y. Gong, K. Okitsu, T. Ozawa, Y. Hawakawa, T. Yamaguchi and M. Kumagawa, Crystal Res. Technol. 27 (1992) 609.

[6] Landolt-Börnstein, New Series, Group III, Vol. 17d (Springer, Berlin, 1984) p. 14.

[7] V.L. Indenbom, Kristall Tech. 14 (1979) 493.

[8] G.M. Blom and T.S. Plaskett, J. Electrochem. Soc. 118 (1991) 1831. 